\title{
Philosophiques
}

\section{Phénoménologie politique du voile}

\section{Hourya Bentouhami}

Volume 44, numéro 2, automne 2017

Les nouveaux horizons du féminisme dans la philosophie francophone

URI : https://id.erudit.org/iderudit/1042334ar

DOI : https://doi.org/10.7202/1042334ar

Aller au sommaire du numéro

\section{Éditeur(s)}

Société de philosophie du Québec

ISSN

0316-2923 (imprimé)

1492-1391 (numérique)

Découvrir la revue

Citer cet article

Bentouhami, H. (2017). Phénoménologie politique du voile. Philosophiques, 44(2), 271-284. https://doi.org/10.7202/1042334ar

\section{Résumé de l'article}

On entend par phénoménologie politique la manière dont l'ordre des apparences, la réalité même de ce qui est vu, est déterminée par des rapports de pouvoir reposant sur des logiques de race, de sexe et de classe. Mon objet porte sur la constitution du voile et des femmes musulmanes qui le portent, dans les pays occidentaux et tout particulièrement en France, comme un objet " phobogène ", qui suscite un dégoût à bout d'oeil. Comment expliquer une telle insistance médiatique et politique à vouloir régler l'ordre d'apparition des femmes voilées ? Ma thèse sera de montrer que la laïcité à la française, dans sa nouvelle version, est fondée sur une théorie des apparences largement déterminée par un imaginaire nationaliste de la différence des sexes, par la réactivation d'un orientalisme sexuel et par l'invisibilisation propre au travail du care auquel les femmes musulmanes sont souvent assignées.
Ce document est protégé par la loi sur le droit d'auteur. L’utilisation des services d’Érudit (y compris la reproduction) est assujettie à sa politique d'utilisation que vous pouvez consulter en ligne.

https://apropos.erudit.org/fr/usagers/politique-dutilisation/ 


\title{
Phénoménologie politique du voile
}

\author{
HOURYA BENTOUHAMI \\ Université de Toulouse 2 - Jean Jaurès
}

\begin{abstract}
RÉSUMÉ. - On entend par phénoménologie politique la manière dont l'ordre des apparences, la réalité même de ce qui est vu, est déterminée par des rapports de pouvoir reposant sur des logiques de race, de sexe et de classe. Mon objet porte sur la constitution du voile et des femmes musulmanes qui le portent, dans les pays occidentaux et tout particulièrement en France, comme un objet «phobogène», qui suscite un dégoût à bout d'œil. Comment expliquer une telle insistance médiatique et politique à vouloir régler l'ordre d'apparition des femmes voilées? Ma thèse sera de montrer que la laïcité à la française, dans sa nouvelle version, est fondée sur une théorie des apparences largement déterminée par un imaginaire nationaliste de la différence des sexes, par la réactivation d'un orientalisme sexuel et par l'invisibilisation propre au travail du care auquel les femmes musulmanes sont souvent assignées.
\end{abstract}

\begin{abstract}
By political phenomenology we understand the manner in which the order of appearances, the reality itself of what is seen, is determined by the relations of power that rest on the logics of race, sex and class. My aim [in this paper] concerns the constitution of the veil and of Muslim women who wear it in Western countries, especially in France, as a "phobogenic" object, which arouses disgust at the sight of it. How to explain the insistence of media and political institutions on regulating the order of appearance of veiled women? My thesis will be to show that laicization on the French model, in its most recent incarnation, is founded on a theory of appearances largely determined by a nationalist imaginary of the differences of the sexes, by the reactivation of a sexual orientalism, and by rendering invisible the caring work to which Muslim women are often relegated.
\end{abstract}

«Le monde est fait de l'étoffe même du corps ${ }^{1}$ » nous dit Merleau-Ponty, entendant par là que le monde est cela que nous voyons, et qu'il n'y a pas d'autre accès aux choses mêmes que ce que nous sentons, organisons par le biais de notre corps. La phénoménologie politique renvoie à la manière dont notre géographie intime - le cercle de la familiarité de mon corps - est investie par des politiques, des mesures administratives, préfectorales, lesquelles décident de ce qui peut circuler ou non, de ce qui peut être vu ou non, de ce qui peut travailler ou non, et dans quelles conditions d'apparition: cette géographie intime révèle ainsi ce qu'est un corps entravé, empêché et à l'inverse ce que peut un corps qui dispose de la liberté de se mouvoir et de sentir au sein d'un monde commun. L'idée principale sous-jacente à une phénoménologie politique du racisme est qu'il n'y a pas de perception visible qui ne soit déjà une construction sociale et politique. Il s'agit ici de voir comment la femme voilée est devenue une catégorie monstrueuse du visible,

1. Merleau-Ponty, Maurice, L'œil et l'esprit, Paris, Quadrige, p. I 595.

PHILOSOPHIQUES 44/2 - Automne 2017, p. 271-284 
qui heurte les sensibilités, révulse les yeux et les estomacs, chacun à sa vue se sentant heurté dans le sentiment de sa propre existence: que ce soit dans une salle de cours, dans le train, au restaurant, dans le métro, lors d'une sortie scolaire, sur une plage, etc. Au sein de la République laïque française qui ne cesse de redéfinir sa laïcité en fonction d'un ordre racial des apparences, où ce n'est pas tant les manifestations du religieux que de l'islam qui sont considérées comme répugnantes, le voile fait office d'objet phobogène, qui encombre le champ de vision ordinaire de la nation. La phénoménologie s'intéresse à l'apparaître, et à ce qui dans cet apparaître constitue un évènement de mon corps en tant qu'il est au monde et non pas simplement dans le monde: chacun est un être qui échappe à toute saisie catégorielle, à toute assignation, il est cet excès de sens qui fait que, idéalement, vous ne pouvez être réduit à la somme de vos composantes organiques ${ }^{2}$. Pourtant, ce sont précisément celles-ci qui peuvent opérer dans le racisme comme autant de marqueurs somatiques de votre indignité: peau, cheveux, nez, bouche, sexe. Car l'une des manières qu'a le racisme de fonctionner est de produire des êtres métonymiques réduits à leurs parties.

En l'occurrence, les femmes voilées sont réduites à «des voiles ambulants» selon le terme de la féministe voilée Ndella Paye de Mamans toutes égales ${ }^{3}$. Une position supposément naïve, mais en réalité investie de politique d'inimitié ${ }^{4}$, peut pousser à ce que l'on s'interroge: qu'est-ce qui nous assure que ce sont bien là des êtres humains, et plus spécifiquement encore que ce sont bien des femmes? Cette perspective d'une phénoménologie à l'aune de la racisation genrée déplace la question classique de la perception telle que Descartes par exemple se la posait lorsqu'il se demandait ce qui pouvait lui assurer que les chapeaux et les manteaux qu'il voyait de sa fenêtre ne recouvraient pas "des spectres ou des hommes feints qui ne se remuent que par ressorts ${ }^{5}$ ?". La seule manière de voir que ce sont de vrais hommes, disait-il, c'est que nous en jugeons ainsi, autrement dit que nous

2. Dans la lignée de Merleau-Ponty et de Beauvoir, Iris Marion Young, considère le corps vécu comme un corps en situation qui doit toujours faire face à un environnement qui modèle sa tenue et lui donne sens: dans cette perspective, le corps des femmes est un corps entravé puisque leurs mouvements ne reçoivent pas, comme c'est le cas pour les corps masculins, un encouragement à se déployer et se coordonner de manière fluide. De fait, les femmes auraient tendance selon elle à faire l'expérience, inhibitrice, de leur corps comme d'un corps sans cesse sous le regard, masculin. Voir Marion Young, Iris, «Throwing Like a Girl: A Phenomenology of Feminine Body Comportment, Motility and Spatiality ", dans On Female Body Experience: "Throwing like a girl" and Other Essays. New York, Oxford University Press, 2005 [1990)].

3. Ndella Paye, «Stop! Mon corps ne vous appartient pas", Mediapart, 23 mars 2015. «Mamans toutes égales» est un collectif de mères de familles qui s'est constitué en opposition à la circulaire Châtel de 2012 interdisant aux mères de familles voilées d'accompagner les enfants lors des sorties scolaires.

4. Achille Mbembe, Politiques d'inimitié, Paris, La Découverte, 2016.

5. René Descartes, Deuxième méditation, Méditations métaphysiques. 
devons tout à la puissance de jugement de notre esprit qui permet réellement de percevoir ce que les yeux seuls ne sauraient voir. L'humanité perçue à partir d'un chapeau ambulant est une déduction de l'esprit. Qu'en est-il du voile? Il semblerait que la perception même du voile défie toute rationalité intellectuelle et opère comme un gage de soustraction à une manière toute particulière d'incarner l'humanité selon les tenants d'un certain ordre sexuel et racial, à savoir celle de la féminité.

Le voile serait ce qui nie la féminité et par là-même l'humanité, celle-ci ne pouvant s'incarner que dans le cadre d'une compétence sexuée de l'apparaître: la féminité devant s'engager dans l'ordre de l'hypervisibilité, sans cesse présente à la saisie d'un regard: soumission à la pulsion scopique qui est propre à ce que l'on pourrait appeler une certaine phénoménologie de la rencontre sexuelle. La femme voilée se soustrairait donc, selon ses détracteurs ${ }^{6}$, à un ordre visible de l'arrangement des sexes, et à une forme de performativité du féminin. Le désordre des superficies visibles qu'opérerait le voile serait ainsi révélateur d'une intériorité "anormale»: le refus de la séduction, c'est-à-dire le refus de correspondre à ce que l'on attend d'une femme, à savoir ce qui dans l'agentivité sexuelle est suffisant pour faire croire à un jeu dans la rencontre sexuelle plutôt qu'à une prédation. Ainsi, ce n'est pas tant de ne pas voir les cheveux de ces femmes qui est visé par l'ensemble des législations et mesures prohibitives du voile, que le désir anormal de se soustraire à un certain réseau de signes patriarcal: en l'occurrence, celui de la disponibilité à la rencontre sexuelle par un vêtement non couvrant. Ce non-désir postulé par le voile est considéré comme une aberration de la raison et un outrage à l'érotisme réglé des relations hétérosexuelles. Mettre un voile recouvre aujourd'hui - au mieux une forme de mauvais goût vestimentaire - au pire une insanité, voire une saleté dans une société marquée par ce que d'aucuns ont appelé la nouvelle laïcité7, c'est-à-dire une laïcité qui veut régler l'ordre des apparitions jusque dans l'espace public, alors même qu'elle n'est pas censée s'y appliquer. J'aimerais précisément voir comment cette nouvelle laïcité se définit par rapport à un ordre racial des apparences ${ }^{8}$, qui serait en l'occurrence une spécificité française par rap-

6. Les positions visant les femmes voilées sont nombreuses et recouvrent un large spectre politique qui brise les lignes de fracture gauche-droite.

7. «La nouvelle laïcité » est ainsi avancée dans un rapport établi en 2003 par François Baroin, homme politique de l'Union pour un mouvement populaire (droite). Elle y désigne le fait que la laïcité est désormais menacée par l'islam, qui aurait remplacé l'Église catholique sur ce point, l'islam opérant par ailleurs depuis les années I980 une offensive culturelle selon lui, si bien que désormais la laïcité serait devenue une valeur de droite là où traditionnellement elle était considérée de gauche, celle-ci ayant supposément cédé au communautarisme et renoncé à la défense de la laïcité.

8. Pour une autre approche phénoménologique, voir Alia Al-Saji, «The Racialization of Muslim Veils: a Philosophical Analysis», Philosophy and Social Criticism, 2010, 36 (8), p. $875-902$. 
port à un sécularisme à l'anglo-saxonne qui relèverait davantage d'une théorie des dispositions: preuve en est la manière dont la France insiste sur l'injonction à la discrétion des musulmans, sur le refus de ce qu'elle considère être des signes ostentatoires. Il s'agira de voir comment la forclusion du voile opère à même le réel dans le champ du visible. Les corps des femmes voilées sont littéralement des corps traqués qui obstruent le champ de visibilité, lui-même jamais neutre ni politiquement ni sexuellement: ce sont des corps qui sont considérés comme des empêcheurs de jouir littéralement, de digérer tranquillement ou encore de se baigner tranquillement, de jouer tranquillement (pour les enfants à la crèche). Ce qui est en jeu une fois de plus ici est donc la construction ethnicisante d'une civilité, d'un caractère national dans le champ même de l'imaginaire perceptif, de ce qui peut constituer un horizon du visible. Voici donc les questions que je me poserai: qu'est-ce que percevoir, et plus encore, percevoir un visage, un corps de femme voilée ? En quoi le voile opère-t-il comme un marqueur de répudiation sociale au sein d'une société qui a érigé la laïcité comme sa forme d'enrégimentement de l'ordre social des apparitions? Qui peut apparaître? À quelles conditions et sous quelles modalités? Ces questions sont largement tributaires de l'impensé colonial, et de la réactivation de ce dernier sous la forme d'un orientalisme sexuel, comme le désigne Todd Shepard'. Enfin, j'aimerais insister sur ce qui jusqu'à maintenant n'a jamais été évoqué à propos des femmes voilées: leur possibilité d'apparition au sein de la sphère du travail.

\section{La laïcité et la nationalisation des apparences: la réactivation de la différence sexuelle}

C'est dans le cadre d'une théorie renouvelée de la laïcité que l'on a pu interpréter le port du voile comme un défi, comme l'incapacité à souscrire aux exigences de l'homo duplex ${ }^{10}$, de la séparation entre privé et public, et de leurs conditions respectives d'apparition. De fait, il nous semble qu'il se soit opéré en France une nationalisation des apparences, au sens où le visible est régi par des normes et des impératifs relevant de l'adhésion ostentatoire à une identité nationale, préalablement ethnicisée (à savoir un athéisme et en même temps, aussi paradoxal cela soit-il, une chrétienté réélaborée comme héritage commun incontournable).

Il me semble qu'il y a en France une conception de la laïcité qui repose sur une théorie des apparences ${ }^{11}$ : pourquoi cette laïcité insiste-t-elle autant

9. Todd Shepard, Mâle décolonisation. L'«homme arabe» et la France, de l'indépendance algérienne à la révolution iranienne, Paris, Payot, 2017.

10. L'expression est de Étienne Balibar dans Saeculum. Culture, religion, idéologie, Paris, Galilée, 20I 2.

11. Je remercie Marwan Mohammed pour avoir suggéré cette idée très riche selon laquelle la distinction entre la laïcité à la française et le sécularisme négocié à l'anglo-saxonne 
sur la présentation visible de soi, sur la représentation? En quoi ce qui se donne à voir aux yeux de tous témoignerait d'une attitude, d'une disposition qui confirmerait ou nierait un attachement, voire une aptitude à la laïcité. Le sécularisme à l'anglo-saxonne par exemple ne met pas autant l'accent sur le visible, c'est pourquoi le voile ne fait pas l'objet d'une obsession avec la même intensité et selon la même spécificité qui est celle d'une obstruction du visible. Ce qui est valorisé dans l'attitude des agents, c'est la possibilité de prendre des décisions neutres, au sens d'une impartialité: la neutralité confessionnelle ou ethnique n'est donc pas demandée antérieurement à l'action de l'individu. Le vêtement à lui seul d'ailleurs ne saurait suffire à définir une compétence: citoyenne, professionnelle, associative, etc. À l'inverse, dans une laïcité pensée conjointement à une politique assimilationniste, il y a une précédence du récit de soi - nécessairement particulariste - que l'universel républicain se doit d'effacer pour faire advenir l'égalité indépendamment des appartenances religieuses, ethniques, identitaires. Or, cette manière d'appréhender l'universel à partir de représentations de la dignité politique du citoyen, et à plus forte raison du fonctionnaire, dans une dimension qui est largement sexuée, genrée, classiste et racisée a donné lieu à une forme spécifique de stratégie du visible, typique d'une politique phénoménologique racialisante. Avant d'en expliquer la logique, il faut noter que le postulat d'une telle politique de régulation raciale du visible est qu'il y aurait quelque chose qui, dans l'Islam, encombre la vue, produit un excès de visibilité en rappelant que l'Islam existe sur le territoire national français comme une composante de l'identité française. Cette visibilité demanderait une réglementation en termes de degré, une sorte de mesure de l'intensité de la visibilité: qui irait de la discrétion à l'ostentation qui correspondrait à autant de degrés moraux de gage ou de désaveu républicain, allant de la foi secrète, quiétiste, à la foi prosélyte qui inviterait à la conversion. Est notamment prégnante cette idée que, à la vue ordinaire d'un voile, certains se sentiraient menacés par ce fichu qui les "agresse». C'est pourquoi Électricité de France par exemple suggère à ses employées voilées d'adopter un "petit foulard de couleur» plutôt qu'un "grand foulard gris». Le guide de l'employé de la Poste distingue quant à lui les emplois en contact avec la clientèle, qui supposent la neutralité de l'agent, de ceux situés en "service arrière », dont les titulaires sont libres de porter un signe religieux visible.

Pour saisir cette volonté de gouverner la visibilité de l'Islam, il faut partir des questions suivantes: qu'est-ce que cela fait de voir un voile pour celles et ceux qui sont étrangers au champ de significations et d'adhésion de ce fichu vestimentaire que l'on a tendance à surinvestir religieusement? Les émotions relatées sont souvent la peur, le dégoût, l'incompréhension, la réprobation morale, les autres symptômes physiologiques affectant les capa-

reposerait sur la distinction respective entre l'insistance sur les apparences et l'insistance mise sur les dispositions des agents fonctionnaires de l'État. 
cités organiques sont: l'incapacité même de respirer ${ }^{12}$, les troubles de l'appétit $^{13}$. Certains enseignants ont pu dire qu'ils se sentaient agressés par la vue d'un voile en cours. Il y a cette idée aussi que l'islam est une culture étrangère, que c'est une religion immigrée (et pas simplement d'immigrés), et que le voile est à ce titre un marqueur allogène, non national qui ne peut donc qu'être exclu de la visibilité publique, et de la possibilité de représenter les valeurs de la France ${ }^{14}$. La raison est que le voile serait un stigmate par lequel on se dénonce comme soumise, arriérée, inculte, inapte à la pensée, et aux soins des autres (comme je le montrerai en troisième partie). Dans cette perspective, le voile fonctionne comme une preuve à charge pour les tenants assimilationnistes, au sens où ce qui va constituer mon identité est précisément ce qui sera considéré comme une preuve contre moi: preuve de mon impuissance, de ma soumission à mes pères, mes frères, etc.

Ainsi, dans la lignée de Sara Ahmed qui dit que la blancheur se porte de manière privilégiée en arrière de $\operatorname{soi}^{15}$, au sens où l'on ignore ce qui ne figure pas dans notre champ de perception, et au sens où l'on peut s'oublier soi-même, le fait d'être musulmane "avouée ", c'est-à-dire voilée, s'accompagne d'une précédence du récit national qui nous a toujours déjà définie comme un être incapable, une victime passive qu'il faudrait au mieux sauver, au pire sanctionner, ou encore plus étrangement qu'il faudrait sauver en sanctionnant, comme le montre l'exemple de l'expulsion des filles voilées des écoles.

Qu'est-ce que cela fait alors de porter le voile? On vous voit venir, on vous attend, et littéralement vous finissez vous-même par vous attendre: l'image que l'on a de vous et les contenus fantasmatiques autour du voile vous précèdent et saturent le champ de votre perception au point que vous n'êtes plus capable de venir au monde sous la forme de l'oblique, de la noncorrespondance aux attentes sociales. Il y a donc quelque chose qui relève de l'épreuve de soi dans cette manière d'être toujours auscultée, regardée, et qui serait caractéristique de ce qu'Elsa Dorlin appelle la phénoménologie de la proie $^{16}$ : se sentant constamment traquée, la proie ne cesse de regarder en retour ceux qui la regardent pour prévenir tout geste agressif. En ce sens le réel est tout entier dans cette attention portée à ce qui me rejette, me nie, et peut surgir sous la forme d'une interaction ordinaire: car la violence ordinaire se trouve implicitement autorisée par les procédures législatives, régle-

12. C’est Catherine Kinzler qui évoque la notion de «respiration laïque» lors de son audition par la Délégation sénatoriale aux droits des femmes, "La laïcité garantit-elle l'égalité femmes-hommes? ", Rapport d'information n ${ }^{\circ}$ IOI (20I6-20I7) du 3 novembre 20I6, p. 42.

13. L'allusion renvoie au refus de servir deux clientes voilées par un gérant de restaurant gastronomique à Tremblay-en-France, en Seine-Saint-Denis, le 27 août 20 I6.

14. Voir à ce propos Abdelmalek Sayad, L'immigration ou les paradoxes de l'altérité, 1 . L'illusion du provisoire, Paris, Raisons d'agir, 2006.

15. Sama Ahmed, "Phenomenology of Whiteness », Feminist Theory, 8, 20 I I.

16. Elsa Dorlin, Sans défense, Paris, La Découverte (à paraître octobre 2017). 
mentaires ou administratives si bien que l'attaque peut venir de toutes parts, d'où ce que Fanon considérait comme la constitution même d'un être paranoïaque toujours à l'affût, sur le qui-vive, et qui produit " un épuisement de l'attention" (Dorlin). Ce que fournit la laïcité comme discours politique, c'est un script des sentiments, c'est-à-dire un langage et une prescription de ce que l'on doit ressentir à la vue d'un voile, et qui nourrit, ou du moins légitime en retour, des formes de violence ordinaire envers les femmes voilées, comme cette femme qui s'est vu arracher son voile par une femme blanche hilare en plein cœur d'un centre commercial très fréquenté ${ }^{17}$.

Mais qu'est-ce qui spécifiquement dans la femme voilée heurte le sens commun de la nation française et provoque un tel dégoût et une telle hilarité? On doit à Joan Scott cette idée que la laïcité à la française ${ }^{18}$ est supportée par une théorie des apparences qui relève amplement du fantasme des signes sociaux et de l'érotisation liée à la différence des sexes, comme parangon d'un esprit à la française, si bien que toute personne dérogeant à cette séduction possible montrerait ainsi son hostilité à toute appartenance à ce qui fait la nature française, son côté licencieux qui irait de la galanterie aristocratique jusqu'à la révolution sexuelle de Mai 1968.

Le voile nierait la possibilité de la rencontre sexuelle, de la séduction et donc de l'égalité: il serait en soi non seulement le marqueur d'une inégalité des sexes mais encore d'une inégalité du désir sexuel. Porter le voile ce serait se soustraire au jeu du chat et de la souris, de la chasse fantasmée, ce serait se retrancher dans un espace qui serait inaccessible à la chasse érotique. Vouloir délibérément s'en soustraire, ce serait ainsi refuser l'adhésion à l'idéal égalitaire de la nation française qui repose sur la différence des sexes, elle-même lisible paradoxalement dans ce registre de la chasse. Joan Scott a bien montré comment cette mythification de la séduction comme résolution de la différence sexuelle et réponse à l'inégalité dés-historicise et justifie les inégalités de genre par le recours à une supposée tradition historique qui précède les temps démocratiques et remonterait aux temps aristocratiques. Comme elle le dit, cette idée place les agréments visibles de la sexualité, de la corporéité offerte, dans un cadre qui ne serait pas celui de la loi, dit-elle, mais du rituel: cela ferait partie d'un «style de vie» amoureuse à la française, et plus généralement d'un arrangement des sexes à la française, qui déborde le cadre du scénario strictement sexuel. Le plus étonnant est que ce discours de la libération sexuelle, le discours de l'hétéronormativité des conduites sexuelles et celui de l'érotisation de la domination, entrent

17. L'agression a eu lieu le $\mathrm{I} 5$ décembre $20 \mathrm{I} 5$ au centre commercial des 4 Temps à La Défense, près de Paris. Voir le récit dans le journal Le Parisien [http://www.leparisien.fr/courbevoie-92400/la-defense-agressee-a-cause-de-son-voile-I 5-I 2-20I 5-53750 I I.php].

18. Joan Scott, De l'utilité du genre, Paris, Fayard, 20I2. Voir particulièrement les chapitres «Sécularité ou sexularité ? La laïcité ou l'égalité des sexes 20 Io» et «La séduction, une théorie française». 
en collusion - alors même qu'ils sont contradictoires — dans le cadre d'une rhétorique empruntée au féminisme qui réinsère le port du voile dans le cadre de sa propre histoire d'émancipation du corps des femmes par la libération vestimentaire. Ainsi en est-il par exemple de l'historienne Christine Bard, autrice de Une histoire politique du pantalon, et qui lors de son audition au Sénat ${ }^{19}$ rappelait que les femmes avaient réclamé de porter le pantalon et de laisser respirer le corps en dénudant certaines de ses parties, jusque-là tenues occultes. La référence à la libération de l'entrave vestimentaire instaure rapidement une confusion, qui finit par comprendre cette libération en termes de nécessaire dévoilement.

C'est une vision de la France qui porte en elle la nostalgie d'un passé largement fantasmé sur la galanterie à la française, donc qui repose largement sur une ethnicisation du désir érotique et un réinvestissement érotique de la différence des sexes. Il y aurait donc chez les femmes voilées quelque chose qui heurte la politesse, la bonne civilité et qui mériterait d'être caché, du fait de son abjection. Et de fait on reproche aux femmes voilées ce à quoi on les accule selon une contradiction opportune: on reproche au voile d'effacer, d'invisibiliser les femmes. Scott décrit ainsi ce qui est considéré implicitement par les "conservateurs» comme une incompatibilité sexuelle de l'islam avec la République.

Le voile serait le signe de cette incompatibilité sexuelle. L'islam en général serait inhabitable sexuellement. Pour comprendre cette association entre théorie des apparences, et théorie de l'arrangement de la différence des sexes, il faudrait comprendre comment la laïcité elle-même est devenue un discours de l'égalité des sexes, ce qu'elle n'était pas historiquement comme le révèle Florence Rochefort ${ }^{20}$ : au contraire la distinction de l'Église et de l'État s'est faite selon un pacte de genre qui tenait les femmes en respect. Il faudrait donc plutôt voir comment l'égalité des femmes s'est construite fallacieusement comme un problème de visibilité plutôt que comme un problème politique engageant les rapports sociaux de sexe aussi bien dans le domaine économique, politique que domestique.

À travers ce discours de l'incompatibilité sexuelle de l'islam et de la République se trouve ainsi réactivé le fantasme orientaliste, colonial et viriliste d'arracher le voile. À quoi correspond ce fantasme? Ce n'est plus une femme stricto sensu, mais la figure de l'Ailleurs insaisissable, qui se dérobe. Ce qui ne peut faire l'objet ni d'une énonciation poétique ni d'une sensibilité incarnée. Tout est fait pour que l'idée même d'une chair, d'un corps qui sent, qui étudie, travaille et rêve soit effacée. La femme orientale est incapable de jouir, et pour cela on va de fait l'exclure de tout ce qui peut se rapporter à

19. La Délégation sénatoriale aux droits des femmes, «La laïcité garantit-elle l'égalité femmes-hommes?", Rapport d'information n ${ }^{\circ}$ IOI (20I6-20I7) du 3 novembre 20I6, p. 46.

20. Florence Rochefort (dir), Le pouvoir du genre: laïcités et religions, 1905-2005, Toulouse, Presses universitaires du Mirail, coll. «Le temps du genre», 2007. 
l'exercice digne de cette jouissance: l'idée même d'un plaisir lui est nié, comme celui de faire des achats en famille au centre-ville, de déguster publiquement un repas gastronomique, de profiter du soleil estival sur une plage.

\section{La réactivation de l'orientalisme sexuel}

Le geste de dévoilement des femmes musulmanes a une densité historique: les affaires du voile remontent de fait à l'époque coloniale durant laquelle le voile était un enjeu militaire - notamment en Algérie pour le cas français - de conquête des esprits et des corps indigènes. Le fantasme d'arracher le voile, de pénétrer les intimités, de conquérir le corps des femmes, comme humiliation des hommes est une stratégie patriarcale de conquête militaire sous d'autres formes, et dont le pendant effectif a été la possibilité sans cesse reconduite du viol. L'histoire coloniale, comme le dit Todd Shepard, est une histoire sexuelle, une histoire politique de la sexualitée ${ }^{1}$, de la prédation de l'érotisme. De plus en plus l'historiographie critique s'est saisie de l'histoire du dévoilement des femmes dans la période coloniale en Algérie pour rendre raison de la profondeur historique d'une politique émancipatrice qui passerait par le dévoilement visible, donné en spectacle, des femmes musulmanes. Il est en effet souvent fait référence à ce que l'on a appelé la bataille du voile, et dont les images sont particulièrement éloquentes et décryptées dans Burning the Veil22. Dans cette fameuse bataille on voit des femmes blanches partisanes de l'Algérie française dévoiler d'autres femmes, algériennes, dans une liesse populaire largement orchestrée. Mais on peut penser aussi à tout ce que le discours orientaliste, de l'ordre des représentations a fourni comme lien entre érotisme et femmes voilées, à travers certes les cartes postales pornographiques ${ }^{23}$, mais aussi à partir de ce qui a été constitué comme un mur à abattre par la «civilité coloniale» et qui est précisément, l'ethos du musulman dont le refus de la rencontre, du regard croisé était perçu comme une forme d'insubordination dans sa manière même de ne pas provoquer la rencontre. C'est ce que pose Fanon: «Il n'y a donc pas chez l'Algérien, dans la rue ou sur une route cette conduite de la rencontre intersexuelle que l'on décrit aux niveaux du regard, de la prestance, de la tenue musculaire, des différentes conduites troublées auxquelles nous a habitués la phénoménologie de la rencontre ${ }^{24}$.»

21. Todd Shepard, Mâle décolonisation. L' "homme arabe» et la France, de l'indépendance algérienne à la révolution iranienne, op. cit.

22. Neil MacMaster, Burning the Veil: The Algerian War and the "Emancipation" of Muslim Women 1654-1962, Manchester, New York, Manchester University Press, 20 I I.

23. Voir Christelle Taraud, Mauresques. Femmes orientales dans la photographie coloniale (1860-1910), Paris, Albin Michel, 2003; La prostitution coloniale. Algérie, Tunisie, Maroc (1830-1962), Paris, Payot, 2003.

24. Franz Fanon, "L'Algérie se dévoile ", L'an $V$ de la révolution algérienne, Paris, La Découverte, 20I I [I959], p. 26. 
Quelle est en effet la conception de l'espace public convoquée pour décrire une certaine identité française, un certain ethos français de l'occupation de l'espace public? Cet espace public est défini dans le Dictionnaire critique et interdisciplinaire de la participation - définition convoquée par la délégation sénatoriale aux droits des femmes — de la manière suivante: " tout espace de rencontre, qu'elle soit fortuite et programmée, où l'on peut faire l'expérience de l'Autre et où la différence, même sa propre différence, est protégée par l'anonymat ${ }^{25}$ ». Au vu de cette définition, ce à quoi échoue l'ethos musulman et plus encore celui de la femme voilée serait l'impossibilité de faire l'expérience de l'Autre autrement que sous la forme de la prédation: de fait la supposée soumission sexuelle des femmes voilées semble être l'envers imaginaire de la menace de l'agression sexuelle par les musulmans migrants dans l'espace public de la fête populaire, comme le révèlent les discours à charge contre les migrants le 3 I décembre 2015 à Cologne en Allemagne $^{26}$. De manière générale, dans la terminologie phénoménologique de Lévinas, la femme voilée, en plus de mettre en échec la rencontre sexuelle, manquerait la rencontre éthique puisqu'elle rendrait difficile à son encontre la possibilité pour celui ou celle qui la voit d'être désarmé, interpellé par le visage de l'Autre: le voile serait donc une forme de solipsisme qui annulerait la responsabilité éthique au monde.

Ainsi, le voile serait incapable selon ses détracteurs de prendre soin des femmes, contrairement à ce que les femmes voilées soutiendraient (le foulard les protégerait des agressions sexuelles, des regards insistants, du harcèlement de rue à caractère sexuel). Mais, plus encore, le voile les empêcherait par là même de prendre soin des autres correctement: non seulement il est sous-entendu dans le discours politique ordinaire que les femmes voilées ne savent pas se défendre mais elles constitueraient les autres femmes - non voilées - comme des femmes disponibles sexuellement. Elles mettraient également en danger celles et ceux dont elles cherchent à prendre soin (comme leurs enfants), ce qui en ferait des mauvaises mères, "elles sont alors de mauvaises accompagnatrices ${ }^{27}$ » mais aussi de mauvaises soignantes, pour une raison spécifique que nous permet de comprendre la phénoménologie politique, à savoir qu'elles ne sauraient pas voir, elles manqueraient d'attention pour les autres car seraient attentives à la seule pureté de leur être. En somme, toute leur attention serait investie religieusement par un

25. Ilaria Casillo, "Espace public», Dictionnaire critique et interdisciplinaire de la participation, Paris, GIS Démocratie et participation, 20I3, cité par La Délégation sénatoriale aux droits des femmes, "La laïcité garantit-elle l'égalité femmes-hommes? ", op. cit., p. 42.

26. Voir notamment la prise de position de l'écrivain algérien Kamel Daoud qui considère que les agressions sexuelles de Cologne seraient dues à des migrants musulmans, qui porteraient en eux la maladie sexuelle de l'Islam: celle qui consiste à faire des femmes, et plus encore des femmes blanches, des proies sexuelles. [http://www.lemonde.fr/idees/article/20I 6/0I/3 I/ cologne-lieu-defantasmes_48566 694_3232.html], consulté le 25-07-20I7.

27. Il est fait référence ici à la circulaire Châtel de 20 I 2 qui interdit aux femmes voilées d'accompagner les enfants lors des sorties scolaires. 
souci de soi. Autrement dit, dans ces discours politiques ordinaires, il semblerait que, au refus d'attribuer une agency et un récit de soi qui serait propre aux femmes voilées elles-mêmes, correspond une autre violence: celle du refus progressif de leur attribuer le soin des autres, notamment en public, dans le cadre de ce qui constitue un service public (ou une "mission de service public »), comme le révèle l'affaire Baby Loup ${ }^{28}$. J'aimerais donc m'attacher désormais à la spécificité de l'économie de la visibilité relative au travail du care en tant que celui-ci est pratiqué par des femmes voilées ${ }^{29}$.

\section{Femmes voilées, dénégation du care et du travail affectif: l'assignation «légale» à la domesticité et au travail invisible}

L'approche phénoménologique permet de voir comment le déploiement du corps des femmes voilées est entravé au travail: la prohibition du voile dans le travail du care, ou plus généralement, dans le travail d'attention à la clientèle, produit en effet des corps empêchés, réduits souvent à leur seule voix lorsque ces femmes sont confinées à des tâches d'attention à la clientèle, où leur visibilité ne heurte pas la clientèle. Le voile au sein du travail du care est vécu comme une menace dans le cadre de ces rapports de production spécifiques que sont les rapports de production de soi, c'est-à-dire les rapports de reproduction où l'enjeu est la possibilité même de se maintenir en vie dans la dignité, et de pouvoir se présenter aux autres sous la forme d'une décence, d'une civilité qui laisse à voir que l'on est seul maître de son apparence ${ }^{30}$. On a souvent dit que la manière dont on apparaissait était une construction, quelque chose qui relevait d'une maîtrise, en oubliant que les conditions de la tenue de soi sont traduisibles en termes d'économie politique de conditions matérielles et genrées d'apparition: et qu'elles sont fournies par toutes les petites mains qui assurent la décence et la dignité de notre apparition en public ${ }^{31}$. De fait — déjà

28. En 2008, une employée musulmane de la crèche privée Baby Loup revient voilée de ses congés d'été. La direction décide de la licencier à la suite des refus de cette dernière de retirer son voile: l'employée considérant être victime d'une discrimination, saisit les Prud'hommes. S'ensuit une longue bataille judiciaire qui voit finalement la décision de renvoi entérinée par les différents tribunaux saisis.

29. Cette dimension du travail est souvent oubliée lorsque l'on parle du discours politique prohibitif à l'égard des femmes voilées, alors même que le travail constitue l'une des modalités essentielles de l'être-au-monde: l'une des conséquences de la pénalisation de l'apparition des femmes voilées dans l'espace public du travail étant précisément leur possible assignation à domicile. Sur cet oubli du travail dans la théorie critique, voir Nasima Moujoud, "Métiers domestiques, voile et féminisme. Nouveaux objets, nouvelles ruptures », Hommes et migrations, $\mathrm{n}^{\circ}$ I $300,20 \mathrm{I} 2$, p. 84-94.

30. Sandra Laugier; Pascale Molinier; Patricia Paperman (dir.), Qu'est-ce que le care? Souci des autres, sensibilité, responsabilité, Paris, Payot, 2009.

31. Voir Joan Tronto, Un monde vulnérable. Pour une politique du care, Paris, La Découverte, 2009; et pour une perspective féministe matérialiste du care, voir Silvia Federici, «Reproduction de la force de travail dans l'économie globale: la révolution féministe inachevée », Point Zéro: propagation de la révolution, Paris, Éditions iXe, 20I6, p. I45-178. 
dans la colonisation et jusqu'à aujourd'hui — ces petites mains ont pu être des femmes voilées dont on a pu considérer qu'elles ne savaient pas prendre soin de leur apparence, mais qu'elles étaient qualifiées pour s'occuper des autres et de la préparation de leur apparition en public.

Je vais donc m'attacher ici à analyser l'économie du visible au sein du travail dit affectif pour comprendre comment le voile est constitué aujourd'hui - et ce, depuis peu - comme une incapacité à prendre soin de soi, et surtout, à prendre soin des autres. L'histoire du capitalisme racial repose donc aussi sur une phénoménologie, sur un ordre réglé des apparitions. À une division raciale et sexuelle du travail correspond une division raciale et sexuelle des apparences: l'apparaître étant une forme de travail dont l'organisation trouve également ses racines et son imaginaire dans la colonisation, l'histoire de l'esclavage, et constitue à ce titre l'un des phénomènes les plus anciens de la mondialisation ${ }^{32}$. Les femmes voilées étant assimilées à des étrangères, des immigrées, leur employabilité dépendait aussi largement de la manière dont on disposait à leur propos d'un discours racialisé de leur compétence au travail du soin ou de nettoyage. En somme la construction d'une altérité remarquable à un signe visible peut aussi bien œuvrer à charge ou à décharge pour les femmes voilées: le voile pouvant être considéré comme le signe d'une compétence maternelle ou au contraire comme le signe d'une mauvaise maternité.

En effet, le voile comme marqueur d'appartenance religieuse, comme marqueur confessionnel avait pu jouer - jusque dans des temps relativement récents - comme une forme de certificat visible de compétence dans le domaine des services, notamment de nettoyage: dans le cadre des services où le recrutement peut s'effectuer à partir de l'ethnicisation de compétences professionnelles, la supposée docilité des femmes musulmanes "avouées", c'està-dire voilées, pouvait œuvrer en leur faveur dans le cadre d'un dispositif de travail qui demande précisément une forme d'effacement et d'invisibilité. En ce sens, une conversion opportune de la sémantique - même hasardeuse $\mathrm{du}$ voile en compétence professionnelle subalterne pouvait œuvrer à une forme d'intégration du voile dans l'espace du travail. Il y avait dans le voile quelque chose qui renvoyait à l'enfermement domestique et à l'assignement à la charge maternelle dans le cadre d'une essentialisation d'une supposée culture musulmane prônant la ségrégation sociale et domestique des sexes, et qui était compatible professionnellement avec ce que l'on attendait à la fois

32. Voir Evelyn Nakano Glenn, «Servitude to Service Work: Historical Continuities in the Racial Division of Paid Reproductive Labor ", Signs, vol. I 8, n I, I992, et Evelyn Nakano Glenn, "Cleaning Up/Kept down: A Historical Perspective on Racial Inequality in "Women's Work" ", Stanford Law Review, vol. 43, n 6, I99I, p. I340; Pour le contexte de la colonisation française, voir Nassima Mekaoui "La "Fatma", création du système colonial ? Généalogie d'une figure de la domesticité féminine "indigène" en Algérie (années I900—I962)", communication au colloque, Domestiques et domesticités. Servir un maître de l'Antiquité à nos jours, Université de Grenoble, 25 mars 2016. 
d'une femme dédiée au travail affectif et d'une femme voilée. Cet ensemble de perceptions imaginaires de la femme voilée a pu fonctionner comme une forme de réconfort ou de confirmation de la compétence de ces femmes à s'occuper du ménage, de la cuisine, des enfants, des personnes âgées, autrement dit de tout ce qui avait un rapport avec une dimension de don de soi sacrificiel de la féminité ainsi caractérisée à partir de la naturalisation d'attributs moraux et physiques sexués. Autrement dit, avoir sa femme de ménage voilée, ou une cuisinière voilée dans les cantines scolaires, pouvait être envisageable et ne semblait pas mettre en cause l'ordre racial, social et genré d'apparition: au contraire, il pouvait contribuer à le confirmer dans sa légitimité.

En réalité, depuis l'affaire Baby Loup et le récent avis de la Cour de justice de l'Union européenne sur le port de signes religieux dans l'entreprise $^{33}$, le voile est en train de devenir un signe religieux œuvrant à charge contre les femmes musulmanes ${ }^{34}$. Il n'est souvent autorisé que dans les métiers où les femmes sont structurellement invisibles: soit par leurs horaires qui font qu'elles ne croisent jamais ou peu les usagers, soit par le fait que l'attention à la clientèle se fasse à distance, hors de la vue. De fait, les centres d'appel constituent un véritable refuge pour les femmes voilées, quitte pour certaines à se voir ainsi déclassées au travail. Ce cas est intéressant, car il montre comment ce qui a pour objet l'attention à la clientèle peut, ne passant plus par la visibilité, se soustraire à l'injonction de dévoilement, précisément parce que l'invisibilité technique expulse du champ de l'imaginable que les femmes dédiées à l'attention puissent être françaises et voilées. En creux, on peut donc voir qu'il y a un privilège du non-voile dans ce contexte racialisé des apparitions au travail, et qui ne constitue pas seulement un primat phénoménologique de pouvoir apparaître aux yeux de tous selon son propre récit de soi. C'est bien en termes de conditions matérielles d'existence que se lisent ces privilèges dans le travail affectif entendu au sens large: en termes d'avancement de carrière, d'occupation de poste valorisant (comme le contact avec la clientèle, les relations publiques). On peut certainement comprendre, avec Sara Farris, que l'injonction au dévoilement s'insère dans une logique capitaliste globale qui «demande d'en finir avec l'incongruité

33. Avis de la Cour de justice de l'Union européenne rendu le I 6 mars 2017 à la suite des cas de deux femmes licenciées en France et en Belgique à cause du port du voile. La CJUE a considéré que les entreprises privées ont droit, sous conditions, d'interdire le port du voile. Pour ce faire, il suffit - en autres choses — qu'elles aient précisé dans le règlement intérieur cette interdiction. Voir aussi Stéphanie Hennette-Vauchez et Vincent Valentin, L'affaire Baby Loup ou la nouvelle laïcité, Paris, 20I4, LGDJ; Stéphanie Hennette-Vauchez, "La régulation juridique du fait religieux: comment lire l'interdiction du voile au prisme du critical race feminism ", dans Hourya Bentouhami et Mathias Möschel, Critical Race Theory, Introduction aux textes fondateurs, Paris, Dalloz, 2017 (à paraître).

34. C'est dans le sillon de l'affaire Baby Loup que bien des entreprises comme EDF ou la Poste ont édité des manuels de laïcité, à usage interne, afin de réglementer la visibilité du voile et de tout signe d'appartenance religieuse. 
du corps féminin caché en tant qu'exception à la règle générale selon laquelle $<$ les femmes> devraient circuler comme des "valeurs franches" 35 ". L'idée sous-jacente d'une telle analyse est la suivante: «pour que la féminité opère sous le capitalisme, le corps féminin doit être exposé pour pouvoir circuler sous un "paradigme marchand" 36 ". On pourrait ajouter que ce n'est que récemment que le voile est devenu une entrave à la marchandisation du corps dans les politiques du care et du travail affectif. La construction idéologique du voile, et plus généralement de l'islam, comme interdit d'apparition éclaire ainsi in fine les transformations du processus de marchandisation des femmes non blanches.

\section{Conclusion}

On peut se demander ce qu'il y a de si dangereux dans l'apparition de l'islam, dans son identification visible aux yeux de tous. Hannah Arendt émet une hypothèse à ce propos pour rendre raison de ce qu'il y a de menaçant à apparaître dans l'espace public, aux yeux d'une politique soucieuse du maintien de l'ordre, au sens large. Elle rappelle un épisode de la Rome impériale où le Sénat avait proposé de décréter le port d'un uniforme pour les esclaves afin qu'on les pût distinguer des hommes libres, mais le Sénat se rétracta et considéra la visibilité comme dangereuse, car les esclaves auraient ainsi pu se reconnaître les uns les autres, et ainsi mesurer leur force en vue d'une possible rébellion: la force des esclaves ne leur venant pas alors de leur nombre - au demeurant proportionnellement peu élevé — que de l'apparition publique sous la forme d'une possible communauté. Elle conclut ainsi que "ce que le sûr instinct politique des Romains jugeait dangereux, c'était l'apparence en tant que telle, indépendamment du nombre de gen $s^{37}$ ».

Cependant, en insistant sur la production notamment légale, par le haut, des processus de racisation du visible, je n'ai pas voulu donner l'impression que le racisme échappe au sens commun. Il s'est agi au contraire de montrer comment ce sens commun qui informe les conduites ordinaires, les interactions quotidiennes trouve son contexte d'intelligibilité et de légitimité dans le cadre de politiques de répudiation sociale du voile. Ce sont ces politiques et discours publics qui autorisent des formes que l'on dit de racisme ordinaire comme les insultes, le harcèlement, les molestations, les vexations qui peuvent confiner à la mort sociale, si ce n'était les capacités de résistance que déploient beaucoup de ces femmes, lesquelles malgré tout s'obstinent à apparaître, à encombrer le champ de vision de l'espace civil de la République laïque française.

35. Sara Farris, "Femonationalism and the 'Reserve' Army of Labor Called Migrant Women", History of the Present, 2(2), 201 2, p. I99.

36. Ibidem.

37. Hannah Arendt, Condition de l'homme moderne, Paris, Pocket, I96I, p. 280. 